\title{
NEW PERIODIC ORBITS AND BASINS FOR FAMILIES OF RESONANT NORMAL FORMS
}

\author{
Bernard J. GEURTS ${ }^{1}$ \\ Center for Theoretical Physics, Twente University, P.O. Box 217, 7500 AE Enschede, \\ The Netherlands
}

Received 15 August 1989

\begin{abstract}
A special class of periodic orbits of parameter dependent families of truncated resonant normal forms is constructed. Their existence is shown for arbitrarily large periods. Explicit analytical criteria are derived for constructing the complete basin of attraction of an invariant circle for normal forms for which the eigenvalue of the linearized mapping is not a multiple root of unity. Persistence of these basins is shown for small perturbations of the parameters. Hence, the existence of bounded orbits for all times established, generically.
\end{abstract}

\section{Introduction}

We study smooth mappings of the complex plane onto itself that posses an elliptic fixed point. The eigenvalue of the linearized mapping in the fixed point lies on the unit circle. They can be transformed, through a polynomial coordinate transformation, to so-called truncated resonant normal forms [1, 2]. These normal form mappings posses a symmetry in the sense that they commute with a certain class of rigid rotations. This allows the construction of families of special periodic orbits whose periods are divisors of the order of the resonance, $Q$, i.e. $\lambda^{Q}=1$. Here $\lambda$ is the eigenvalue of the linearized mapping in the fixed point. We show the existence of these special periodic orbits for arbitrarily large periods, which amounts to a new version of the PoincareBirkhoff theorem for generic mappings.

If the eigenvalue is not a multiple root of 1 it can be shown $[2,3]$ that the normal form mapping possesses attracting and repulsing invariant circles on which a rigid rotation is induced. Explicit analytical criteria for constructing the complete basins of attraction or repulsion of these circles are derived. The existence of bounded orbits for all times is established even if the eigenvalue is a multiple root of unity. Hence, the basins are extended to so called $Q$ -

\footnotetext{
${ }^{1}$ New address: Philips Natuurkundig Laboratorium, Postbus 80000, 5600 JA Eindhoven, The Netherlands.
} 
resonant normal forms, that is to normal forms for mappings where $\lambda^{Q}=1$ for some integer $Q$.

Mappings of the plane onto itself have been widely used for modelling complex dynamical behavior in a great variety of physical systems [4]. Since we consider the local dynamics of such mappings in a neighborhood of an elliptic fixed point, we generally deal with the generic situation around periodic states in systems where dissipation is small. As an example, one may think of vortices in a "nearly" Newtonian fluid and their persistence under small perturbations of the system.

In section 2, we derive the truncated normal form mapping, and show that it commutes with a class of rigid rotations. Based on this we construct families of periodic orbits, and show existence of special periodic orbits whose periods are divisors of the order of the resonance $Q$. Then in section 3 , we first consider truncated normal forms where the eigenvalue is not a multiple root of unity. It will be shown that these mappings possess attracting and repulsing invariant circles, and explicit anaytical criteria for constructing the complete basin of attraction or repulsion are derived. Then, these results are extended to normal forms where the eigenvalue is a multiple root of unity showing the existence of bounded orbits for all times, generally, for small perturbations.

\section{Invariance of the dynamics and new families of periodic orbits for truncated resonant normal forms}

We consider families of smooth mappings $P_{\mu}$ that depend smoothly on a parameter vector $\mu \in \mathbb{R}^{k}$, and for which 0 is an elliptic fixed point. The theory of the reduction of a family of mappings to normal form [1-3] furnishes a local polynomial coordinate tranformation such that $P_{\mu}$ can be written as

$$
\begin{aligned}
P_{\mu}(z)= & z\left[1+f\left(|z|^{2} ; \mu\right)\right] \mathrm{e}^{2 \pi \mathrm{i} g\left(|z|^{2} ; \mu\right)}+\sum_{3 \leqslant l Q+2 j \leqslant L+1} d_{j l}(\mu)|z|^{2 j} \bar{z}^{l Q-1} \\
& +\mathcal{O}\left(|z|^{2 L+1}\right)
\end{aligned}
$$

provided the eigenvalue of $\mathrm{D} P_{\mu}(0)$ is such that $\lambda^{Q}=1$, for some integer $Q$, the order of the resonance. In this equation $L$ is the order of the truncation and it is assumed that $Q \leqslant L$. Furthermore, $f$ and $g$ are polynomials of degree $L$, with coefficients that depend smoothly on $\mu$, and the $d_{j l}$ are smooth functions of $\mu$ [2]. The complete mapping will be referred to as the $Q$-resonant normal form, and the terms in the summation of (2.1) as $Q$-resonant terms. If $\lambda$ is not a multiple root of unity for $Q \leqslant L$, or if we disregard the $Q$-resonant terms, (2.1) reduces to

$$
P_{\mu}(z)=z\left[1+f\left(|z|^{2} ; \mu\right)\right] \mathrm{e}^{2 \pi \mathrm{i} g\left(|z|^{2} ; \mu\right)}+\mathcal{O}\left(|z|^{2 L+1}\right) .
$$


This mapping has a trivial dynamics; it maps circles onto circles, and leaves circles with radii $\xi$ such that $f(\xi ; \mu)=0$ or -2 , invariant.

For convenience we will express the normal form mapping in polar coordinates. This allows to study to some extent the angular and radial part of the mapping separately. Denoting the mapping expressed in polar coordinates $(\rho, \theta)$ by $\tau_{\mu}$, and identifying a point $z \in \mathbb{C}$ by $z=\rho^{1 / 2} \mathrm{e}^{2 \pi \mathrm{i} \theta}$, one readily obtains

$$
\tau_{\mu}(\rho, \theta)=\left(D^{2}(\rho, \theta ; \mu) \rho, \theta+g(\rho ; \mu)+E(\rho, \theta ; \mu)\right),
$$

where

$$
\begin{aligned}
& D(\rho, \theta ; \mu)=\frac{[1+f(\rho ; \mu)]+\Omega_{1}(\rho, \theta ; \mu)}{\cos [2 \pi E(\rho, \theta ; \mu)]}, \\
& E(\rho, \theta ; \mu)=\frac{1}{2 \pi} \arctan \left(\frac{\Omega_{2}(\rho, \theta ; \mu)}{[1+f(\rho ; \mu)]+\Omega_{1}(\rho, \theta ; \mu)}\right)
\end{aligned}
$$

and

$$
\begin{aligned}
& \Omega_{1}(\rho, \theta ; \mu)=\sum_{j, l} d_{j l}(\mu) \rho^{(l Q+j-1) / 2} \cos \{2 \pi[l Q \theta+g(\rho ; \mu)]\}, \\
& \Omega_{2}(\rho, \theta ; \mu)=\sum_{j, l} d_{j l}(\mu) \rho^{(l Q+j-1) / 2} \sin \{2 \pi[l Q \theta+g(\rho ; \mu)]\} .
\end{aligned}
$$

For the mapping (2.2) this simplifies to

$$
\tau_{\mu}(\rho, \theta)=\left([1+f(\rho ; \mu)]^{2} \rho, \theta+g(\rho ; \mu)\right) .
$$

Although the expression for $\tau_{\mu}$ is quite complicated, it possesses a very important property; it commutes with the family of rigid rotations $R_{(m / Q)}$, i.e.,

$$
\tau_{\mu} \circ R_{(m / Q)}(\rho, \theta)=R_{(m / Q)}{ }^{\circ} \tau_{\mu}(\rho, \theta),
$$

where $R_{(m / Q)}(\rho, \theta)=(\rho, \theta+m / Q), m \in \mathbb{Z}$. In words, the orbit with initial point $(\rho, \theta+n / Q)$ can be obtained from the orbit with initial point $(\rho, \theta)$ by shifting all angles by an amount $n / Q$; the dynamics is invariant under $R_{(m / Q)}$. Hence we can state that if $\Gamma\left(\rho_{1}, \theta_{1}\right)$ is a linearly stable (unstable) periodic orbit of period $q$, and winding number $p / q$, then all orbits $\Gamma\left(\rho_{1}, \theta_{1}+n / Q\right), n=$ $1,2, \ldots, Q-1$, are linearly stable (unstable) periodic orbits of the same type, with the same eigenvalues for the linearized mapping around them.

Periodic orbits that share the symmetry of the mapping, i.e. their periods are divisors of $Q$, can be characterized more accurately. Indeed, if the period $q$ is a divisor of $Q$, and if there is a point $\left(\rho_{1}, \theta_{1}\right)$ such that $D^{2}\left(\rho_{1}, \theta_{1} ; \mu\right)=1$, 
$g\left(\rho_{1} ; \mu\right)+E\left(\rho_{1}, \theta_{1} ; \mu\right)=p / q$, then $\Gamma\left(\rho_{1}, \theta_{1}\right)$ is a periodic orbit of period $q$, and winding number $p / q$, and

$$
\begin{aligned}
& \pi_{1}\left(\tau_{\mu}^{j}\left(\rho_{1}, \theta_{1}\right)\right)=\rho_{1}, \\
& \pi_{2}\left(\tau_{\mu}^{j}\left(\rho_{1}, \theta_{1}\right)\right)=\theta+\frac{j p}{q},
\end{aligned}
$$

where $\pi_{l}$ is the projection on the $l$ th coordinate. So if $D^{2}=1, g+E=p / q$, the symmetry-sharing periodic orbits exist, and have constant radial part and constant angle increment $p / q$. For a subclass of $Q$-resonant normal forms, the condition $D^{2}=1, g+E=p / q$ can be shown to be necessary and sufficient for $\Gamma\left(\rho_{1}, \theta_{1}\right)$ to be a symmetry-sharing periodic orbit.

Let us represent the parameter vector $\mu$ by $\left(\mu_{1}, \mu_{2}\right)$, and suppose that at $\left(\mu_{1}, 0\right)$ the mapping reduces to $(2.6)$. Then if there is a $\xi \geqslant 0$ such that

$$
\begin{aligned}
& {\left[1+f\left(\xi ; \mu_{1}\right)\right]^{2}=1, \quad g\left(\xi ; \mu_{1}\right)=p / q,} \\
& \partial_{\xi} g\left(\xi ; \mu_{1}\right) \neq 0, \quad D_{\mu_{1}} g\left(\xi ; \mu_{1}\right) \text { is invertible }
\end{aligned}
$$

then there exists an open neighborhood $U$ of $\left(\mu_{1}, 0\right)$ such that for all $\left(\mu_{1}, \mu_{2}\right) \in \mathrm{U}$, there exists a pair $\left(\rho_{1}, \theta_{1}\right)$ such that $D^{2}\left(\rho_{1}, \theta_{1} ; \mu_{1}, \mu_{2}\right)=1$, $g\left(\rho_{1} ; \mu_{1}\right)+E\left(\rho_{1}, \theta_{1} ; \mu_{1}, \mu_{2}\right)=p / q$. The argumentation is standard and rests on the implicit function theorem. It is similar to that given by Chenciner [2]. For symmetry-sharing periodic orbits, the above statement amounts to an existence proof for resonant normal forms. Also, from the conservation of the Poincaré index [5] one infers that all symmetry sharing periodic orbits, as long as they exist, have the form expressed by (2.8). Apart from that, we point out that the above existence proof is valid for symmetry-sharing periodic orbits of arbitrarily large period [6].

We have shown that, due to the commutation of the resonant normal form mapping with a family of rigid rotations, the existence of one periodic orbit implies that a whole family of the same type periodic orbits exists, related to the first by a rotation over $2 \pi n / Q$. For symmetry-sharing periodic orbits this property could be used to show existence for arbitrarily large periods, and to show that those orbits have constant radial part and constant angle increment.

\section{Invariant circles and boundedness of orbits}

In this section we consider the basic problem of the boundedness of orbits for resonant normal forms. It will be shown briefly that (2.6) possesses 
invariant circles that are, generically, either attracting or repelling. Explicit analytical expressions for determining the complete basin of attraction or repulsion will be derived, and the existence of attracting invariant circles of (2.6) will be related to boundedness of orbits for full $Q$-resonant normal forms, even if the invariant circle has "broken up" due to the variation of the parameters.

We first turn to the normal form of type (2.6). Considering the radial part of the mapping one clearly recognizes that if

$$
f(\xi ; \mu)=0 \quad(\text { or }-2), \quad \xi \geqslant 0,
$$

then the mapping possesses an invariant circle with radius $\sqrt{\xi}$. On this circle the mapping induces a rigid rotation, yielding orbits with winding number $g(\xi, \mu)$. These invariant circles are, generically, attracting or repulsing, corresponding to the fact whether or not the radial part of the mapping is a contraction on a neighborhood of $\xi$. Introducing the short hand notation

$$
F(\rho ; \mu)=[1+f(\rho ; \mu)]^{2}
$$

and $\mathrm{C}_{\xi}=\left\{\left.z \in \mathbb{C}|| z\right|^{2}=\xi\right\}$, we have the following local explicit characterization of attracting or repelling invariant circles:

(i) if $-2<\xi \partial_{\rho} F(\xi ; \mu)<0$ then $\mathrm{C}_{\xi}$ is attracting,

(ii) if $-2>\xi \partial_{\rho} F(\xi ; \mu)$ or $\partial_{\rho} F(\xi ; \mu)>0$ then $C_{\xi}$ is repelling,

where $\partial_{\rho} F$ is the partial derivative of $F$ with respect to $\rho$.

Based on this local characterization we can construct criteria for determining the full basin of attraction or repulsion of the invariant circles. For convenience we introduce the following notation:

$$
\Delta_{\xi}\left(\epsilon_{1}, \epsilon_{2}\right)=\left\{\rho \in \mathbb{R}^{+} \mid 0<\xi-\epsilon_{1} \leqslant \rho<\xi+\epsilon_{2}\right\}
$$

First we turn to repelling invariant circles. One easily verifies that

(a) if $\partial_{\rho} F(\xi ; \mu)>0$, and $\epsilon_{1}, \epsilon_{2}$ are such that

$$
\begin{array}{ll}
F(\rho ; \mu)<1 & \text { for } \xi-\epsilon_{1}<\rho<\xi, \\
F(\rho ; \mu)>1 & \text { for } \xi<\rho<\xi+\epsilon_{2},
\end{array}
$$

or

(b) if $-2>\xi \partial_{\rho} F(\xi ; \mu)$, and $\epsilon_{1}, \epsilon_{2}$ are such that 


$$
\begin{aligned}
& F(\rho ; \mu)>\frac{2 \xi-\rho}{\rho} \text { for } \xi-\epsilon_{1}<\rho<\xi, \\
& F(\rho ; \mu)<\frac{2 \xi-\rho}{\rho} \text { for } \xi<\rho<\xi+\epsilon_{2},
\end{aligned}
$$

then the radial part of $\tau_{\mu}$ is not a contraction on $\Delta_{\xi}\left(\epsilon_{1}, \epsilon_{2}\right)$, and $C_{\xi}$ is a repelling invariant circle with basin of repulsion equal to $\Delta_{\xi}\left(\epsilon_{1}, \epsilon_{2}\right)$ and its preimages under iterants of $\tau_{\mu}$.

Likewise, the global analogue of (i) for attracting invariant circles can be expressed as follows:

if $-2<\xi \partial_{\rho} F(\xi ; \mu)<0$, and $\epsilon_{1}, \epsilon_{2}$ are such that

$$
\begin{aligned}
& 1<F(\rho ; \mu)<\frac{2 \xi-\rho}{\rho} \text { for } \xi-\epsilon_{1}<\rho<\xi, \\
& \frac{2 \xi-\rho}{\rho}<F(\rho ; \mu)<1 \text { for } \xi<\rho<\xi+\epsilon_{2},
\end{aligned}
$$

then $C_{\xi}$ is an attracting invariant circle with basin of attraction $\Delta_{\xi}\left(\epsilon_{1}, \epsilon_{2}\right)$ and its preimages under iterants of $\tau_{\mu}$. In addition, we notice that if $-2<$ $\xi \partial_{\rho} F(\xi ; \mu)<-1$ then all orbits in the basin converge asymptotically alternating to $\xi$ under $\pi_{1}\left(\tau_{\mu}(.,).\right)$ and if $-1<\xi \partial_{\rho} F(\xi ; \mu)<0$ then all orbits in the basin converge asymptotically monotonously to $\xi$ under $\pi_{1}\left(\tau_{\mu}(.,).\right)$.

The relatively simple dynamics of (2.6) will now be used to relate the existence of attracting invariant circles of (2.6) to boundedness of orbits of the full $Q$-resonant normal forms. We will establish this property of generic structural stability [1] by using a general boundedness theorem. This theorem will first be prepared and then the boundedness of orbits established.

Let us introduce the minimal and maximal bounding mappings associated with a smooth mapping $G: \mathbb{R}^{n} \rightarrow \mathbb{R}^{n}$ by

$$
\begin{aligned}
& R_{G, \min }(\alpha)=\min _{x \in C_{\alpha}}\|G(x)\|^{2}, \\
& R_{G, \max }(\alpha)=\max _{x \in C_{\alpha}}\|G(x)\|^{2},
\end{aligned}
$$

where $C_{\alpha}$ is a sphere in $\mathbb{R}^{n}$ with radius $\sqrt{\alpha}$. $G$ will be called order preserving if for all $\alpha \geqslant 0$, and all $x \in G^{j}\left(C_{\alpha}\right)$,

$$
R_{G, \min }^{j}(\alpha) \leqslant\|x\|^{2} \leqslant R_{G, \max }^{j}(\alpha) .
$$

It is straightforward to show that $G$ is order preserving if and only if $R_{G, \min }$, 
$R_{G \text {, max }}$ are increasing. If $G$ is order preserving then the $j$ th image of $C_{\alpha}$ under $G$ is completely enclosed by the spheres with radii $R_{G, \min }^{j}(\alpha)$ and $R_{G, \max }^{j}(\alpha)$. Hence, if $R_{G, \min }$ has an attracting fixed point $\xi_{\min }$, and $R_{G, \max }$ an attracting fixed point $\xi_{\max }$, and if $\alpha$ lies in the basin of $\xi_{\min }$ and $\xi_{\max }$ under $R_{G \text {, min }}$ and $R_{G, \max }$ respectively, then all orbits with initial point $x \in C_{\alpha}$ are bounded and

$$
\xi_{\min } \leqslant \lim _{j \rightarrow \infty}\left\|G^{j}(x)\right\|^{2} \leqslant \xi_{\max }
$$

The above theorem is quite powerful since it allows to consider two onedimensional boundedness problems, instead of the full $n$-dimensional boundedness problem.

We will now apply the above theorem to the boundedness of orbits for full $Q$-resonant normal forms which are sufficiently close to (2.6). In general one may write, expressing again the parameter vector $\mu$ as $\left(\mu_{1}, \mu_{2}\right)$,

$$
\begin{aligned}
& R_{\mu_{1}, \mu_{2}, \min }(\alpha)=\chi_{1}\left(\mu_{1}, \mu_{2}, \alpha\right) F\left(\alpha ; \mu_{1}\right) \alpha, \\
& R_{\mu_{1}, \mu_{2}, \max }(\alpha)=\chi_{2}\left(\mu_{1}, \mu_{2}, \alpha\right) F\left(\alpha ; \mu_{1}\right) \alpha,
\end{aligned}
$$

where $\chi_{1}, \chi_{2}$ are smooth functions of $\mu_{1}, \mu_{2}$ and $\alpha$ for $\left(\mu_{1}, \mu_{2}\right)$ in a neighbourhood of $(0,0), \chi_{1}\left(\mu_{1}, 0, \alpha\right)=1=\chi_{2}\left(\mu_{1}, 0, \alpha\right)$. One then easily verifies that if $-1<\xi \partial_{\rho} F\left(\xi ; \mu_{1}\right)<0$ and $f\left(\xi ; \mu_{1}\right)=0$ (or -2 ), then there exists an open neighborhood $U$ of $\left(\mu_{1}, 0\right)$ and an $\epsilon>0$ such that for all $z \in \mathbb{C}$, with $\|\left. z\right|^{2}-$ $\xi \mid<\epsilon$, and all $\left(\mu_{1}, \mu_{2}\right) \in \mathrm{U}$, the orbits with $z$ as initial point are bounded. The proof of this statement reduces to a verification of the fact that $R_{\mu_{1}, \mu_{2} \text {, min }}$ and $R_{\mu_{1}, \mu_{2} \text {, max }}$ are increasing on a neighborhood of $\xi$, and have attracting fixed points $\xi_{\min }, \xi_{\max }$ respectively. Since the basins of $\xi_{\min }$ under $R_{\mu_{1}, \mu_{2}, \min }$, and of $\xi_{\max }$ under $R_{\mu_{1}, \mu_{2}, \max }$ are overlapping, and include a neighborhood of $\xi$, application of the above boundedness theorem completes the proof.

Hence we have shown the persistence of the basin of attraction of an invariant circle of a normal form of type (2.6), if $Q$-resonant terms are added. This implies boundedness of nearby orbits, even if the invariant circle itself has "broken up" under the variation of the parameters, e.g. if a periodic orbit were induced by (2.6) on that circle.

\section{Concluding remarks}

We have shown that $Q$-resonant normal form mappings possess a $Q$-fold symmetry, in the sense that they commute with the rigid rotations $R_{(m / Q)}$, 
where $Q$ is the order of the resonance. This property allows the construction of families of periodic orbits, if one is known. Also it gives the option of constructing simple symmetry-sharing periodic orbits, and show the existence for arbitrarily large periods. This is of importance since periodic orbits are very useful in investigating the dynamics of nonlinear mappings. They also furnish approximants of more complex orbits of the mapping such as invariant curves and attracting objects.

Furthermore we have shown that, generically, the boundedness of orbits is not affected by small perturbations of the mapping parameters, provided $Q$-resonant terms are small. The existence of bounded orbits for all times can be of great importance in studying for instance of stability/long-time behavior of solutions of (systems of) ordinary differential equations, and also in many technical applications.

\section{Acknowledgements}

I would like to thank Dr. R. Martini, Dr. F. Twilt and Dr. P. Jonker of the Department of Applied Mathematics of the University of Twente for several useful discussions. Furthermore, I would like to thank Dr. R.H.G. Helleman of the Department of Applied Physics of the University of Twente for his support and kind interest in the problems.

\section{References}

[1] V.I. Arnol'd, Geometrical Methods in the Theory of Ordinary Differential Equations (Springer, New York, 1983).

[2] A. Chenciner, Bifurcations de diffeomorphismes de $\mathbf{R}^{2}$ au voisinage d'un point fixe elliptique, in: Les Houches, Session 23, G. Ioos, R. Helleman and D. Stora, eds. (North-Holland, Amsterdam, 1981).

[3] A. Chenciner, Bifurcations de points fixes elliptique, I. Courbes invariant, Extrait des publications Mathematiques de l'IHES, no. 61 (1984).

[4] A.J. Lichtenberg and M.A. Lieberman, Regular and Stochastic Motion (Springer, New York, 1982).

[5] J. Greene, R.S. MacKay, F. Vivaldi and M. Feigenbaum, Physica D 3 (1981) 468.

[6] A. Chenciner, Topologie differentiele sur un enonce dissipatif du theoreme geometrique de Poincaré-Birkhoff, C.R. Acad. Sci. Paris, Ser. I 21 (1983) 243. 